\title{
A giant leiomyoma of the breast
}

\author{
Spasimir Todorov Shopov \\ From Pathologist, Department of Pathology, MBAL “Uni Hospital” Ltd., Panagyurishte, Bulgaria
}

\begin{abstract}
Breast leiomyoma is a rare benign tumor arising from the nipple and/or areola or from smooth muscle metaplasia of myoepithelial or myofibroblast cells. Despite its benign morphology, breast leiomyoma can create diagnostic confusion. Here, we report a rare case of a single leiomyoma of the breast in a 52-year-old woman. The patient reported a lump in her right breast for 1 year, and in the past 6 months, it has grown in size. Physical examination showed a dense mass in the right breast, without axillary lymphadenomegaly. Excisional biopsy revealed a well-defined cell tumor by intertwining the spindle cell folds with fibrillar and eosinophilic cytoplasm. Histopathological and immunohistochemical studies help to discriminate between leiomyoma and other benign and malignant breast lesions. Her results are discussed in our report.
\end{abstract}

Key words: Breast, Diagnosis, Leiomyoma, Tumor

$\mathrm{L}$ eiomyoma of the breast is one of the rarest benign mesenchymal tumors and represents no more than $1 \%$ of all breast neoplasms [1]. It occurs more frequently in the subareolar region because of the presence of the greater amount of smooth muscle [1]. Leiomyoma of the breast parenchyma in the absence of periareolar lesions is uncommon. To date, 33 cases of parenchymal leiomyoma of the breast have been reported [2,3]. As the initial diagnosis, a needle biopsy reveals a benign mesenchymal lesion, but the dilemma between leiomyoma and fibromatosis remains.

Here, we report a rare case of a single leiomyoma of the breast in a 52-year-old woman. Our report presents the $34^{\text {th }}$ consecutive case and documents the results of mammography and histopathology.

\section{CASE REPORT}

A 52-year-old woman was admitted with a history of a lump in her right breast for 1 year. She also reported that in the past 6 months, the lump had increased and sometimes hurt a little. The patient reported two normal pregnancies ending in two live births without miscarriage. There was no familial burden of breast cancer. Previous prophylactic mammograms have found no pathological changes in the mammary glands.

Clinically, a dense tumor formation was palpated in the upper lateral quadrant of the right mammary gland with dimensions

\section{Access this article online}

Received - 31 October 2021

Initial Review - 16 November 2021

Accepted - 25 November 2021

DOI: $10.32677 /$ ijcr.v7i12.3154 of about $8 \mathrm{~cm}$, which was located $3 \mathrm{~cm}$ from the areola. The formation was slightly fixed in-depth, but not with the overlying skin. There was no change in the color of the overlying skin and pathological secretion from the nipple. No enlarged axillary lymph nodes or changes in the left mammary gland were found.

A mammogram revealed a dense, oval, and non-calcified lesion. There appeared no axillary lymphadenopathy. Routine blood tests were within normal limits. Analyzing the clinical and mammographic findings, a thick needle biopsy was performed to reveal the histogenesis of the tumor. Histologically, spindle cells without atypia were found, which were arranged in fascicles. There was no necrosis, mitotic activity. Immunohistochemical study showed diffuse, intense staining for vimentin and desmin. The proliferative activity detected by Ki 67 showed single positive nuclei below $2 \%$. Based on the results, leiomyoma or possibly breast fibromatosis was diagnosed.

After 10 days, a quadrantectomy was performed on the right breast. The tumor was sent for pathological examination in our department. Macroscopically, the part of the parenchyma of the breast with the overlying skin is shown in Fig. 1a. The cutting surface, a densely elastic tumor formation of $8 \mathrm{~cm}$ (Fig. 1b) in diameter with a uniform whitish slightly nodular cut surface (Fig. 1c), was seen. The tumor does not infiltrate the overlying skin.

Microscopically, hematoxylin and eosin (H and E) examination demonstrated spindle cells without atypia, which were arranged in fascicles. They were missing necrosis, mitotic activity, or vascular invasion (Fig. 2a and b). The immunohistochemical study indicated diffuse, intense staining for desmin (Fig. 3a) and

Correspondence to: Spasimir Todorov Shopov, Department of Pathology, MBAL "Uni Hospital” Ltd., str. "Georgi Benkovski” 100, 4500 Panagyurishte, Bulgaria. E-mail: sshopov1@abv.bg

(C) 2021 Creative Commons Attribution-NonCommercial 4.0 International License (CC BY-NC-ND 4.0). 


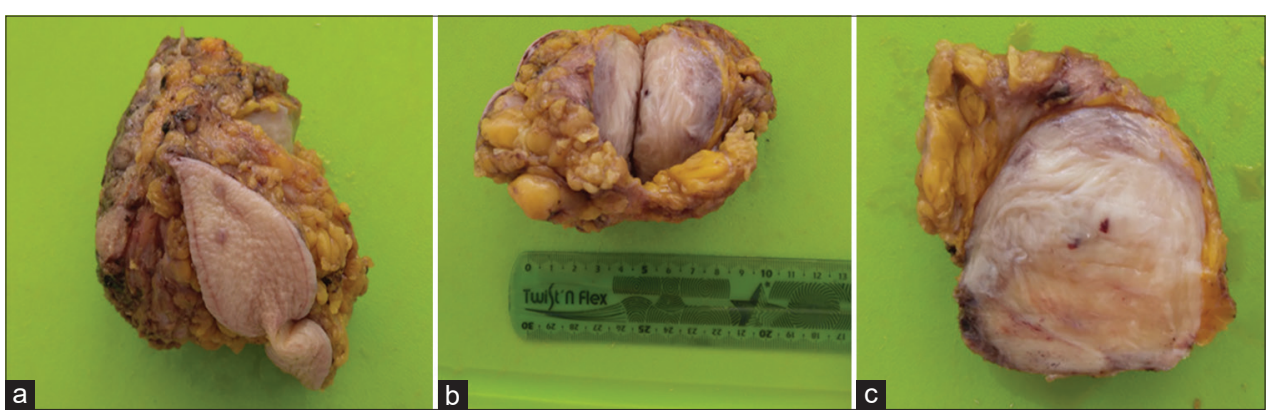

Figure 1: Macroscopic picture. (a) Part of the breast parenchyma with overlying skin after a quadrantectomy; (b) Tumor $8 \mathrm{~cm}$ in diameter; (c) Cut surface: White, well demarcated but non-encapsulated tumor, with swirling and visible area from where a biopsy was taken

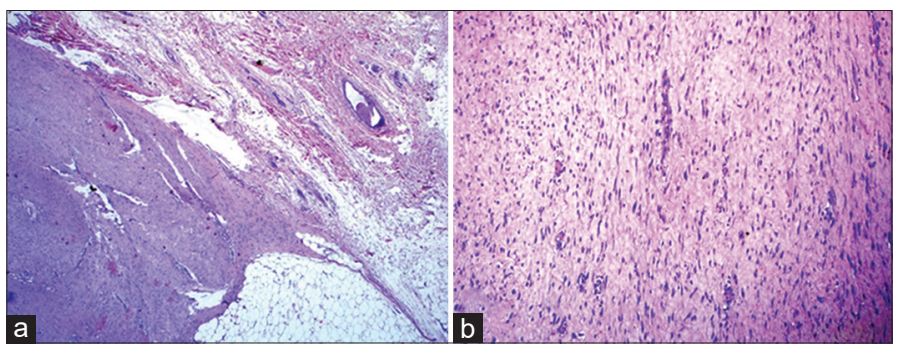

Figure 2: Histology (H and E): (a) Well-demarcated tumor lesion composed of spindle cells without atypia, which was arranged in fascicles. Visible several mammary ductules near the tumor (enlargement $\times 25$ ); (b) Fascicles of monotonous spindle cells with indistinct borders, eosinophilic cytoplasm, some of the nuclei with rounded and others with tapered ends. They are missing necroses, mitotic activity, or vascular invasion (enlargement $\times 50$ )

weakly for smooth muscle actin (SMA) (Fig. 3b). The proliferative activity was below 1\% with Ki67 (Fig. 3c). CD 34 as well as the Verhoeff Van Gieson histochemical study for collagen fibers were negative. Based on the results, intraparenchymal leiomyoma of the breast was diagnosed. At follow-up examinations at 1 and 6 months, there were no subjective complaints from the patient. No pain on examination and local recurrence were found. Routine blood counts were within normal limits. Ultrasound of both breasts showed no pathological changes.

\section{DISCUSSION}

The first description of parenchymal leiomyoma of the breast was made by Strong in 1913 [4]. Since then, no more than 33 cases have been published $[2,3]$. We report the $34^{\text {th }}$ case of breast leiomyoma, applying a series of macroscopic imaging to routine mammography and histological examination for the $1^{\text {st }}$ time. Leiomyoma of the breast is a rare benign non-epithelial tumor. Due to the abundance of smooth muscle cells around the nipple and areola, breast leiomyoma is the most common in these areas, with no difference between the two sexes [5].

Depending on the origin of the tissues, breast leiomyomas can be conditionally divided into two types: Superficial (from the smooth muscles of the skin) and vascular (from the vascular endothelium). Superficial leiomyomas are located in the skin and subcutaneous tissues and involve the nipple or areolar region, while vascular leiomyomas are located deeper within the breast parenchyma and are less common than the superficial leiomyomas [6].
The histogenesis of these tumors is still controversial and unknown, although there are various theories trying to explain the origin of these neoplasms. The theory of Kaufman and Hirsch suggests that they originate from the myocytes around the capillaries in the subcutaneous tissue of the mammary gland [5]. According to Diaz-Arias et al., a proliferation of smooth muscle cells surrounding blood vessels, teratoid origin with the extreme overgrowth of myomatous elements, differentiation of multipotent mesenchymal cells in breast tissue, and derivation from myoepithelial cells of breast ducts with clear differentiation to smooth muscle, or embryologically displaced smooth muscle cells from the nipple can be the possible sources for the origin of such neoplasms [7].

Comparing symptoms and clinical appearance, except for a clinically palpable painless lump, patients mostly complained of discomfort and pain. Tumor sizes varied from 0.5 to $13.0 \mathrm{~cm}$ and most of them were well-circumscribed. Most cases were treated by quadrantectomy, only Boscaino et al. reported two cases of recurrence that was initially diagnosed as leiomyomas. They have been revised and higher mitotic activity has been found, which includes borderline leiomyomas and possibly leiomyosarcomas [8].

On mammography, the majority of reported leiomyomas are well marginated, homogenous, and dense [6]. The histopathological properties of all leiomyomas show groups of interlacing bundles of spindle-shaped cells with vesicular nuclei and eosinophilic cytoplasm, with no or infrequent mitosis [5]. Immunohistochemical staining for this condition indicates positivity for vimentin, desmin, and SMA [7], and the proliferative activity with Ki67 is extremely low.

The case we reported had a typical mammographic image of a well-defined lesion in the right breast-upper outer quadrant. Due to the history of rapid growth in the past 6 months, a core biopsy was performed, which showed a benign non-epithelial lesion positive for vimentin, desmin, weakly for SMA, and extremely low proliferative activity. This necessitated a quadrantectomy without lymph dissection. Despite the characteristic macroscopic image well presented in the report, is needed differential diagnosis with leiomyosarcoma, fibromatosis, phyllodes tumor, adenoleiomyoma, fibroadenoma with prominent smooth muscle, benign spindle cell tumor of the breast, fibrous histiocytoma, myoid hamartoma, and myoepithelioma [2]. Among these, the most important one is 


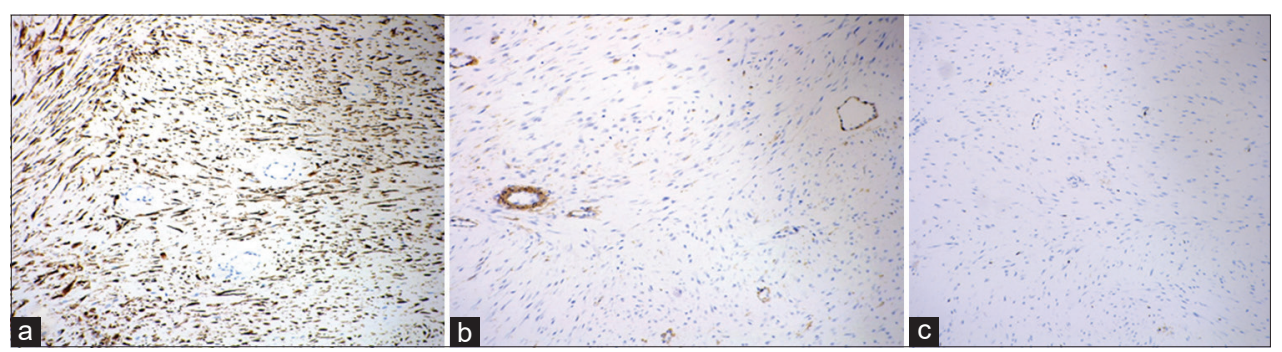

Figure 3: Immunohistochemistry: (a) Positive expression of desmin (enlargement $\times 100$ ); (b) Smooth muscle actin weakly positive (enlargement $\times 50)$; (c) Ki 67 not more than $1 \%($ enlargement $\times 100)$

leiomyosarcoma, and in our case fibromatosis, due to the weak positivity of SMA. The distinction from leiomyosarcoma is important since leiomyosarcomas may show local recurrence or distant metastasis by hematogenous route long after excision. The leiomyosarcoma feature marked cellular atypia, high grade of mitosis (2-16 mitotic figures per 10 high-power fields), atypical mitoses, vascular invasion, and necrosis [3], which is not observed in the case we report. Despite the weak positivity of SMA, we ruled out fibromatosis with a Verhoeff Van Gieson histochemical test that showed no red positive for collagen fibers. Hamartoma lacked fatty and epithelial elements. Phyllodes tumor shows well demarcated with a leaf-like pattern. Epithelial component with intracanalicular model and subepithelial condensation with increased stromal cellularity adjacent to the epithelium. There are no atypia and polymorphism in benign representatives. In the case we report, there was no epithelial component. Fibroadenoma is a welldefined biphasic tumor with a stromal and glandular component. The difference with the case we report is the lack of a glandular component in our case. A benign fibrous histiocytoma is with a prominent storiform pattern of uniform spindle cells with illdefined eosinophilic cytoplasm and bland, elongated, or plump vesicular nuclei with no atypia often hemangiopericytomalike vasculature, scattered lymphocytes, either multinucleated giant cells or foam cells. The histological pattern, as well as the negative CD34 in the case we reported, did not support benign fibrous histiocytoma. Significant in the reported case is the presentation of a large intraparenchymal leiomyoma located in the usual site for breast cancer. Its well-illustrated macroscopic appearance and poor positivity of SMA, with positivity for vimentin, desmin, and extremely low proliferative activity. Here, we present the benefits of a forgotten histochemical test - Verhoeff Van Gieson.

\section{CONCLUSION}

We report an extremely rare manifestation of intraparenchymal leiomyoma of the breast. The clinical entity is like other benign tumors. The mammography is characteristically benign, which helps rule out the hypothesis of cancer. In histopathological evaluations, it is important to make a differential diagnosis with leiomyosarcoma. The standard recommended treatment is quadrantectomy with free margins. There is no risk of local recurrence.

\section{REFERENCES}

1. Kafadar MT, Yalçın M, Gök MA, Aktaş A, Yürekli TS, Arslan Aİ. Intraparenchymal leiomyoma of the breast: A rare location for an infrequent tumor. Eur J Breast Health 2017;13:156-8.

2. Miroslav G, Radovic MS, Darko Z, Nebojsa I, Dejan N, Dragan R, et al. Intraparenchimal leiomyoma of the breast. Arch Iran Med 2015;18:1-5.

3. Brandão RG, Elias S, Nazário AC, Assunção MD, Papa CC, Facina G. Leiomyoma of the breast parenchyma: A case report and review of the literature. Sao Paulo Med J 2018;136:177-81.

4. Strong LW. Leiomyoma of the breast. Am J Obstet 1913;68:53-5.

5. Kaufman HL, Hirsch EF. Leiomyoma of the breast. J Surg Oncol 1996;62:62-4.

6. Heyer H, Ohlinger R, Schimming A, Schwesinger G, Grunwald S. Parenchymal leiomyoma of the breast-clinical, sonographic, mammographic and histological features. Ultraschall Med 2006;27:55-8.

7. Diaz-Arias AA, Hurt MA, Loy TS, Seeger RM, Bickel JT. Leiomyoma of the breast. Hum Pathol 1989;20:396-9.

8. Boscaino A, Ferrara G, Orabona P, Donofrio V, Staibano S, de Rosa G. Smooth muscle tumors of the breast: Clinicopathologic features of two cases. Tumori 1994;80:241-5.

Funding: None; Conflicts of Interest: None Stated.

How to cite this article: Shopov ST. A giant leiomyoma of the breast. Indian J Case Reports. 2021;7(12):516-518. 